\title{
Undamped Oscillations of Collisionless Stellar Systems: Spheres, Spheroids and Discs
}

\author{
S. Sridhar* \& R. Nityananda Raman, Research Institute, Bangalore 560080 \\ Received 1989 February 2; accepted 1989 June 7
}

\begin{abstract}
The collisionless Boltzmann equation governing self-gravitating systems such as galaxies has recently been shown to admit exact oscillating solutions with planar and spherical symmetry. The relation of the spherically symmetric solutions to the Virial theorem, as well as generalizations to non-uniform spheres, uniform spheroids and discs form the subject of this paper. These models generalize known families of static solutions. The case of the spheroid is worked out in some detail. Quasiperiodic as well as chaotic time variation of the two axes is demonstrated by studying the surface of section for the associated Hamiltonian system with two degrees of freedom. The relation to earlier work and possible implications for the general problem of collisionless relaxation in self gravitating systems are also discussed.
\end{abstract}

Key words: stellar dynamics—galaxies

\section{Introduction}

In a recent paper, Sridhar (1989) - henceforth Paper 1-has shown that the collisionless Boltzmann equation (CBE) which is used to describe stellar systems has fully selfconsistent oscillatory solutions. The general strategy is to choose uniform density models with a time-dependent gravitational potential quadratic in the space coordinates. The phase-space density $f(x, v, t)$ (mass per unit phase volume) of the model is chosen to be a function of integrals of motion in this potential, thereby automatically satisfying the CBE by Jeans' theorem (see e.g. the text by Binney \& Tremaine 1987).

An integral due to Lewis (1968) for the time-dependent harmonic oscillator plays the same role for these oscillating models as energy does for the corresponding static models. Apart from Lewis' original paper, Goldstein's (1980) well-known text gives a nice discussion of the Lewis invariant. The existence of such an invariant follows from the linearity of the equations of motion of the oscillator. A set of points in phase space lying on an ellipse will continue to do so after a linear transformation of $x$ and $v$. An invariant quadratic in $x$ and $v$ with time-dependent coefficients must therefore exist.

This paper first makes explicit the relation between the Virial theorem and the spherical systems constructed in Paper 1. We then go on to construct oscillating spheroidal solutions of the CBE. In Section 2 we discuss the consistency of the general

* also Joint Astronomy Programme, Indian Institute of Science, Bangalore 560012 
spherical model with the Virial theorem. A particular "cold" model with easily visualized dynamics is given in Section 3. Steady-state uniform-density spheroids are generalized to oscillating models by the replacement of energies by the corresponding Lewis invariants in Section 4. In contrast to the spherical case there is now a pair of coupled equations for the oscillations of the $a_{1}$ and $a_{3}$ axes of the spheroid. Section 5 gives the behaviour of the solutions, using the Poincare surface of section for the Hamiltonian system with two degrees of freedom which governs the evolution of $a_{1}$ and $a_{3}$. Section 6 gives our conclusions and some implications of these result in the more general context of time-dependent behaviour in collisionless gravitating systems.

\section{The virial theorem and the time-dependent spherical models}

In Paper 1 we showed how to construct time-dependent uniform-density spheres starting from the distribution function describing static spheres. The time dependence of the radius was shown to be governed by a simple differential equation (Equation 22, Paper 1)

$$
\ddot{\xi}+\frac{\sqrt{\omega_{0}}}{\xi^{2}}-\frac{1}{\xi^{3}}=0
$$

where $\xi$ is proportional to the radius and $\omega_{0}$ is a constant. In this section we derive Equation (1) from the Virial theorem. Apart from being a rederivation, the calculations make explicit the physical significance of the terms entering (1).

We start with the distribution function of a static uniform-density sphere written in the form (Equation 13, Paper 1)

$$
f_{0}=f\left(E / \omega_{0}, L^{2} ; \omega_{0}, R_{0}\right)
$$

The mass $\left(M_{0}\right)$, potential energy $\left(W_{0}\right)$ and the kinetic energy $\left(T_{0}\right)$ are easily calculated for a uniform sphere of radius $R_{0}$

$$
M_{0}=\frac{\omega_{0}^{2} R_{0}^{3}}{G}, \quad W_{0}=-\frac{3}{5} \frac{\omega_{0}^{4} R_{0}^{5}}{G}, \quad T_{0}=-\frac{W_{0}}{2} .
$$

The time-dependent sphere is constructed by replacing $E / \omega_{0}$ in (2) by $\mathscr{I}$ :

$$
f=f\left(\mathscr{I}, L^{2} ; \omega_{0}, R_{0}\right)
$$

where

$$
\mathscr{I}=\frac{r^{2}}{2 \xi^{2}}+\frac{1}{2}|\xi \mathbf{v}-\xi \mathbf{r}|^{2} .
$$

Direct calculations in the time-dependent case based on (3) and (4) yield

$$
\begin{aligned}
& \text { mass }=M=M_{0}, \\
& \text { radius }=R=\sqrt{\omega_{0}} \zeta R_{0}, \\
& \text { potential energy }=W=W_{0} / \xi \cdot \sqrt{\omega_{0}}, \\
& \text { mean velocity }=\mathrm{v}_{\mathrm{m}}(\mathrm{r}, t)=(\xi / \xi) \mathrm{r}, \\
& \text { bulk kinetic energy }=T_{b}=\int \rho \frac{v_{\mathrm{m}}^{2}}{2} \mathrm{~d}^{3} r=\left(\frac{\xi}{\omega_{0}}\right)^{2} T_{\mathrm{o}} .
\end{aligned}
$$


heat (kinetic energy associated with local peculiar velocities) $=T_{\mathrm{h}}=\frac{1}{2} \int \mid \mathrm{v}-$ $\left.v_{\mathrm{m}}\right|^{2} f \mathrm{~d}^{3} v d^{3} r=T_{0} / \omega_{0} \xi^{2}$

total kinetic energy $=T=T_{b}+T_{h}$,

$$
I=\int \rho r^{2} \mathrm{~d}^{3} r=\frac{2 \xi^{2}}{\omega_{0}}
$$

The Virial theorem is

$$
\frac{1}{2} \ddot{I}=2 T+W .
$$

Using the formulas for $I, T_{b}, T_{h}$ and $W$ from (5) in (6), we see that

$$
\ddot{\xi}+\sqrt{\omega_{0}} / \xi^{2}-1 / \xi^{3}=0
$$

which is what we set out to verify. The term $\left(-1 / \xi^{3}\right)$ resembles the centrifugal term occurring in the radial equation for a central force problem and is clearly proportional to $T_{\mathrm{h}} / \xi$; it makes good physical sense that the system is hottest when it is maximally compressed and that the heat makes the system bounce back. The total energy

$$
E=T+W=\frac{2 T_{0}}{\omega_{0}}\left(\frac{\xi^{2}}{2}-\frac{\sqrt{\omega_{0}}}{\xi}+\frac{1}{2 \xi^{2}}\right) .
$$

Equation (1) admits a first integral

Therefore

$$
\lambda=\frac{\xi^{2}}{2}-\frac{\sqrt{\omega_{0}}}{\xi}+\frac{1}{2 \xi^{2}} .
$$

$$
E=\frac{2 T_{0}}{\omega_{0}} \lambda .
$$

When $\lambda \geqslant 0$, the solutions to (1) are unbounded as $t \rightarrow \infty$, while for $\lambda<0$, the solutions are bounded and time periodic. From (9) we see that these correspond to positive and negative total energies respectively.

\section{Models made up of nonintersecting spherical shells}

Paper 1 described a general approach using which oscillating generalizations of uniform density spherical models could be constructed. One of the special cases mentioned there was a uniform density sphere made up entirely of particles on circular orbits, i.e. having minimum energy for a given angular momentum. This model is radially "cold", i.e. there is a unique value (zero) of $v_{r}$, the radial component of the velocity at a given position $\mathbf{r}$. The corresponding oscillating model is obtained by populating those orbits which have the lowest possible value of the Lewis invariant for a given value of the angular momentum. At a given radius $r$, this is achieved by setting

$$
\frac{\mathrm{d} r}{\mathrm{~d} t}=v_{r}=\frac{\xi}{\xi} r
$$

(this implies $r(t) \propto \xi(t)$ for a given particle). The model is still cold radially. In other words, the particles can be grouped into spherical shells which oscillate without 
crossing. This special class of models is worth a separate discussion because it admits of a straightforward physical interpretation and an interesting generalization.

To visualize the particle motions, consider a spherical shell made up of particles in elliptical Kepler orbits with a fixed period and eccentricity, all starting with the same orbital phase, e.g. apocentre. Let the tangential velocities be isotropically distributed so that all possible orbital planes are equally represented. The shell will clearly pulsate with its size obeying the radial equation of the Kepler problem (since $\xi \propto r$ and $\xi$ obeys the radial Kepler equation - see Equation 1).For self consistency, we need to maintain the Kepler motion by providing a spherically symmetric mass distribution with fixed mass within this shell (no crossings). This can clearly be done by more such shells, pulsating similarly and in phase with the first with the same period and eccentricity. The constant period implies $M(<a) \propto a^{3}$ where $a$ is the semi-major axis. This implies uniform density, which can be achieved by a suitable choice of masses and radii of the shells.

The physical interpretation of the radially cold uniform density model suggests the following generalization. Let the different shells still oscillate in phase with the same period. The eccentricity $e$ is now allowed to vary from shell to shell in such a manner as to avoid crossings. The distribution of mass with respect to the semimajor axis $a$ is the same as before, but the density is clearly nonuniform because $r$ is a nonlinear function of $\alpha$ (e.g. at apocentre)

$$
r_{>}=a(1+e(a))
$$

\section{Oscillations of homogeneous spheroids}

We use the method presented in Paper 1 to study oscillations of uniform-density spheroids. The method is applicable because the interior potential of a homogeneous spheroid is quadratic in the spatial coordinates. A spheroid with axes $\left(a_{1}, a_{2}, a_{3}\right.$ with $a_{1}=a_{2}$ ) along the $x, y$ and $\mathrm{z}$ directions respectively and mass density $\rho_{0}$ has an interior potential (see e.g. Chandrasekhar 1969).

$$
\varphi=\Omega_{0}^{2} \frac{r^{2}}{2}+\omega_{0}^{2} \frac{z^{2}}{2} ; \quad r^{2}=x^{2}+y^{2}
$$

where

axis ratio

$$
\begin{aligned}
\Omega_{0}^{2} & =2 \pi G \rho_{0} A_{1}(m), \\
\omega_{0}^{2} & =2 \pi G \rho_{0} A_{3}(m), \\
m & =a_{3} / a_{1},
\end{aligned}
$$

$$
\begin{aligned}
A_{1}(m) & =\left(\frac{m}{1-m^{2}}\right) h(m)+1-\frac{1}{1-m^{2}}, \\
h(m) & =\sin ^{-1}\left(\sqrt{1-m^{2}}\right) / \sqrt{1-m^{2}} ; \quad m<1, \\
& =\ln \left(m+\sqrt{m^{2}-1}\right) / \sqrt{m^{2}-1} ; \quad m>1 .
\end{aligned}
$$

Since $\varphi$ and $\rho_{0}$ satisfy $\nabla^{2} \varphi=4 \pi G \rho_{0}$, we have the following relation between $A_{1}$ and $A_{3}$ :

$$
A_{3}(m)=2-2 A_{1}(m)
$$


Some examples of phase-space distribution functions for static uniform spheroids are Freeman's rotating spheroid (or the related nonrotating version due to Zel'dovich and Bisnovatyi-Kogan) which is cold in the plane of rotation and Polyachenko's hot spheroid (see e.g. Fridman and Polyachenko (FP), 1984 for details and references).

We first explain the general strategy and then go to details. The distribution functions of these models depend on the following integrals of motion

$$
\begin{aligned}
E_{\perp} & =\frac{v_{x}^{2}}{2}+\frac{v_{y}^{2}}{2}+\Omega_{0}^{2} \frac{r^{2}}{2}, \\
E_{z} & =\frac{v_{z}^{2}}{2}+\omega_{0}^{2} \frac{z^{2}}{2}, \\
L & =x v_{y}-y v_{x}
\end{aligned}
$$

The time-dependent model is constructed from the static distribution function by replacing $E_{\perp} / \Omega_{0}$ and $E_{Z} / \omega_{0}$ by the corresponding Lewis invariants (see Equation 19). The axes are proportional to $\xi(t)$ and $\eta(t)$ (see Equation 24) which from the construction of the Lewis invariants satisfy Equations (21). Self-consistency eliminates the explicit time dependence in these equations and yields a pair of autonomous, coupled, second order, ordinary differential equations for $\xi$ and $\eta$ (Equation 26).

Uniform spheroids are characterized by at least three parameters determining the mass $M$, axes $a_{1}, a_{3}$. For convenience we choose the parameters to be $\Omega_{0}, \omega_{0}, a_{1}$. Then, the density $p_{0}$ is determined from Poisson's equation.

$$
4 \pi G \rho_{0}=2 \Omega_{0}^{2}+\omega_{0}^{2}
$$

while $a_{3}$ is determined by Equation (11)

$$
A_{1}\left(a_{3} / a_{1}\right)=\Omega_{0}^{2} / 2 \pi G \rho_{0}
$$

and

$$
M=\frac{4 \pi}{3} \rho_{0} a_{1}^{2} a_{3}
$$

We write the distribution function for any static uniform spheroid in the form

$$
f_{0}=f\left(\frac{E_{\perp}}{\Omega_{0}}, \frac{E_{z}}{\omega_{0}}, L ; \Omega_{0}, \omega_{0}, a_{1}\right) .
$$

The density $\quad \rho_{0}=\int f_{0} \mathrm{~d}^{3} v=\nabla^{2} \varphi / 4 \pi G$.

Therefore,

$$
\begin{aligned}
\int f\left(\frac{v_{x}^{2}}{2 \Omega_{0}}\right. & \left.+\frac{v_{y}^{2}}{2 \Omega_{0}}+\Omega_{0} \frac{r^{2}}{2}, \frac{v_{z}^{2}}{2 \omega_{0}}+\omega_{0} \frac{z^{2}}{2}, x v_{y}-y v_{x} ; \Omega_{0}, \omega_{0}, a_{1}\right) \mathrm{d}^{3} v \\
& =\left(2 \Omega_{0}^{2}+\omega_{0}^{2}\right) / 4 \pi G \quad \text { when } \quad \frac{r^{2}}{a_{1}^{2}}+\frac{z^{2}}{a_{3}^{2}} \leqslant 1=0 \quad \text { otherwise }
\end{aligned}
$$

Defining

$$
\begin{gathered}
X=\sqrt{\Omega_{0}} x, \quad Y=\sqrt{\Omega_{0}} y, \quad Z=\sqrt{\omega_{0}} z \\
U_{X}=v_{x} / \sqrt{\Omega_{0}}, \quad U_{Y}=v_{y} / \sqrt{\Omega_{0}}, \quad U_{Z}=v_{z} / \sqrt{\omega_{0}},
\end{gathered}
$$


we have

$$
\begin{gathered}
\int f\left(\frac{U_{X}^{2}}{2}+\frac{U_{Y}^{2}}{2}+\frac{X^{2}+Y^{2}}{2}, \frac{U_{Z}^{2}}{2}+\frac{Z^{2}}{2}, X U_{Y}-Y U_{X} ; \Omega_{0}, \omega_{0}, a_{1}\right) \mathrm{d}^{3} U \\
=\left(2 \Omega_{0}^{2}+\omega_{0}^{2}\right) / 4 \pi G \Omega_{0} \sqrt{\omega_{0}} \text { when } \frac{X^{2}+Y^{2}}{\Omega_{0} a_{1}^{2}}+\frac{Z^{2}}{\omega_{0} a_{3}^{2}} \leqslant 1 \\
=0 \text { otherwise. }
\end{gathered}
$$

The time-dependent model is constructed from the static distribution function by replacing $E_{\perp} / \Omega_{0}$ and $E_{\mathrm{z}} / \omega_{0}$ respectively by the corresponding Lewis invariants (see Paper 1)

$$
\begin{gathered}
I_{1}=\frac{r^{2}}{2 \xi^{2}}+\frac{1}{2}\left|\xi \mathbf{v}_{\perp}-\xi \xi \mathbf{r}\right|^{2} ; \quad \mathbf{r}=(x, y, 0) ; \quad \mathbf{v}_{\perp}=\left(v_{x}, v_{y}, 0\right), \\
I_{3}=\frac{z^{2}}{2 \eta^{2}}+\frac{1}{2}\left(\eta v_{z}-\dot{\eta} z\right)^{2} .
\end{gathered}
$$

The interior potential is

$$
\varphi(r, z, t)=\Omega^{2}(t) \frac{r^{2}}{2}+\omega^{2}(t) \frac{z^{2}}{2}
$$

where $\Omega^{2}(t)$ and $\omega^{2}(t)$ are determined from the density $p(r, z, t)$ and the time-dependent axis ratio by formulas similar to Equation (11).

$\xi, \eta$ are any solutions to

$$
\begin{array}{ll}
\ddot{\xi}+\Omega^{2}(t) \xi-1 / \xi^{3}=0 ; & \xi>0, \\
\ddot{\eta}+\omega^{2}(t) \eta-1 / \eta^{3}=0 ; & \eta>0 .
\end{array}
$$

The time-dependent distribution function is

$$
f=f\left(I_{1}, I_{3}, L ; \Omega_{0}, \omega_{0}, a_{1}\right) .
$$

The density is

$$
\rho=\int f \mathrm{~d}^{3} v
$$

Therefore

$$
\rho=\int f\left(\frac{r^{2}}{2 \xi^{2}}+\frac{1}{2}\left|\xi \mathbf{v}_{\perp}-\xi \mathbf{r}\right|^{2}, \frac{z^{2}}{2 \eta^{2}}+\frac{1}{2}\left(\eta v_{z}-\dot{\eta} z\right)^{2}, x v_{y}-y v_{x} ; \Omega_{0}, \omega_{0}, a_{1}\right) \mathrm{d}^{3} v .
$$

Defining

$$
\begin{gathered}
X^{\prime}=x / \xi, \quad Y^{\prime}=y / \xi, \quad Z^{\prime}=z / \eta, \\
U_{X}^{\prime}=\xi v_{x}, \quad U_{Y}^{\prime}=\xi v_{y}, \quad U_{Z}^{\prime}=\eta v_{z}, \\
\rho=\frac{1}{\xi^{2} \eta} \int f\left(\frac{X^{\prime 2}+Y^{\prime 2}}{2}+\frac{U_{X}^{\prime 2}+U_{Y}^{\prime 2}}{2}, \frac{Z^{\prime 2}}{2}+\frac{U_{Z}^{\prime 2}}{2}, X^{\prime} U_{Y}^{\prime}-Y^{\prime} U_{X}^{\prime} ; \Omega_{0}, \omega_{0}, a_{1}\right) \mathrm{d}^{3} U .
\end{gathered}
$$


From (18) and (22)

$$
\begin{aligned}
\rho & =\frac{1}{\xi^{2} \eta} \frac{\left(2 \Omega_{0}^{2}+\omega_{0}^{2}\right)}{4 \pi G \Omega_{0} \sqrt{\omega_{0}}} \text { when } \frac{X^{\prime 2}+Y^{\prime 2}}{\Omega_{0} a_{1}^{2}}+\frac{Z^{\prime 2}}{\omega_{0} a_{3}^{2}} \leqslant 1 \\
& =0 \text { otherwise. }
\end{aligned}
$$

Therefore the new axes are

$$
\begin{aligned}
& b_{1}=b_{2}=\sqrt{\Omega_{0}} a_{1} \xi, \\
& b_{3}=\sqrt{\omega_{0}} a_{3} \eta,
\end{aligned}
$$

and the axis ratio $u=b_{3} / b_{1}$.

From (11)

$$
\begin{aligned}
& \Omega^{2}=2 \pi G \rho A_{1}(u), \\
& \omega^{2}=2 \pi G \rho A_{3}(u) .
\end{aligned}
$$

Writing $C=\frac{2 \Omega_{0}^{2}+\omega_{0}^{2}}{2 \Omega_{0} \sqrt{\omega_{0}}}, K=\sqrt{\frac{\omega_{0}}{\Omega_{0}}} \frac{a_{3}}{a_{1}}$, and using (23),(24) and (25) in Equations (21) we get the following equations governing the behaviour of the axes of the homogeneous spheroid

$$
\begin{aligned}
& \ddot{\xi}+\frac{C}{\xi \eta} A_{1}\left(\frac{K \eta}{\xi}\right)-\frac{1}{\xi^{3}}=0, \\
& \ddot{\eta}+\frac{C}{\xi^{2}} A_{3}\left(\frac{K \eta}{\xi}\right)-\frac{1}{\eta^{3}}=0 .
\end{aligned}
$$

\section{Some properties of the oscillations of uniform spheroids}

\subsection{Hamiltonian Formulation}

In this section we point out some general features of solutions to (26). Equations (26) can be recast in Hamiltonian form by a simple scaling.

$$
x=\xi, \quad y=K \eta .
$$

The axes

$$
b_{1}=\sqrt{\Omega_{0}} a_{1} x, \quad b_{3}=\sqrt{\Omega_{0}} a_{1} y,
$$

while the axis ratio (ellipticity) is

Introducing a function

$$
u=b_{3} / b_{1}=y / x
$$

$$
g(u)=\frac{h(u)-u}{1-u^{2}}
$$


we can express $A_{1}$ and $A_{3}$ in terms of $g$ :

$$
\begin{aligned}
& A_{1}(u)=u g(u), \\
& A_{3}(u)=2-2 u g(u) .
\end{aligned}
$$

For convenience we shall use constants $\alpha$ and $\beta$ in place of $C$ and $K$

$$
\begin{gathered}
\alpha=K^{4}=\frac{\omega_{0}^{2}}{\Omega_{0}^{2}}\left(\frac{a_{3}}{a_{1}}\right)^{4}, \\
\beta=C K=\sqrt{\Omega_{0}} / g\left(a_{3} / a_{1}\right), \\
0<\alpha, \quad \beta<\infty .
\end{gathered}
$$

Written in terms of $x, y, \alpha$ and $\beta$, Equation (26) reads

$$
\begin{array}{r}
\ddot{x}+\frac{\beta}{x^{2}} g-\frac{1}{x^{3}}=0, \\
\ddot{y}+\frac{2 \beta}{x^{2}}(1-u g)-\frac{\alpha}{y^{3}}=0 .
\end{array}
$$

To see that (33) can be derived from a Hamiltonian, we need the following identity for $g$

$$
\left(1-u^{2}\right) \frac{\mathrm{d} g}{\mathrm{~d} u}=3 u g-2 .
$$

Using (34) it is easy to verify that (33) is generated by the Hamiltonian (with parameters $\alpha$ and $\beta$ in the potential $\mathscr{V}$ )

$$
\mathscr{H}=\frac{p_{x}^{2}}{2}+p_{y}^{2}+\mathscr{V}(x, y)
$$

Where

$$
\mathscr{V}=\frac{1}{2 x^{2}}+\frac{\alpha}{4 y^{2}}-\frac{\beta}{x}\left[u-\left(1-u^{2}\right) g\right] .
$$

An immediate consequence is that is a constant of motion Direct calculations using the Virial theorem and (33) show that

$$
\mathscr{H}=\frac{5 E}{2 M \Omega_{0} a_{1}^{2}}
$$

where $\mathrm{M}$ and $E$ are total mass and energy of the model. The time-independent Hamiltonian structure of (33) guarantees that oscillations of these uniform spheroids do not damp asymptotically. Another advantage is that we can use Poincare's method of the surface of section (see e.g. Lichtenberg \& Lieberman 1983) to understand the nature of these oscillations.

For every allowed value of $\alpha$ and $\beta, \mathscr{V}$ has a minimum whose location $\left(x_{0}, \mathrm{y}_{0}\right.$ is determined by $\partial \mathscr{V} / \partial x=\partial \mathscr{V} / \partial y=0$. Equivalently with $u=\mathrm{y}_{0} / x_{0}$,

$$
\begin{aligned}
& \alpha=2 u_{0}^{3}\left(1-u_{0} g\left(u_{0}\right)\right) / g\left(u_{0}\right), \\
& \beta=\frac{1}{x_{0} g\left(u_{0}\right)} .
\end{aligned}
$$


The Solutions are unique; $(\alpha, \beta) \Leftrightarrow\left(\mathrm{x}_{0}, y_{0}\right)$. The minimum in $\mathscr{V}$ corresponds to a static spheroid with axis ratio $u_{0}$. From (37) it is clear that $u_{0}$ depends only on $\alpha$ implying that $\alpha$ alone determines the ellipticity of the underlying static model (while $\alpha$ is dimensionless, $\beta$ has dimensions of(time $\left.)^{1 / 2}\right), u_{0}$ is an increasing function of $\alpha$ implying that prolateness of the static model increases with increasing $\alpha$. For $\alpha=1$, the static model is a sphere. Away from the minimum and toward the coordinate axes, $\mathscr{V}$ rises indefinitely. Fig. 1 shows a contour plot of $\mathscr{V}$ for $\alpha=1$ and $\beta=3 / 2$. The minimum is at $x_{0}=1, y_{\mathrm{o}}=1$ where $\mathscr{V}=-0.75$. The contours of $\mathscr{V}$ for other values of $\alpha$ and $\beta$ are topologically similar. The value of $\mathscr{V}$ at $\left(x_{0}, y_{0}\right)$ is

$$
\mathscr{V}_{0}=\frac{\beta^{2} g}{2}\left[u_{0}^{2} g-g-u_{0}\right]<0 .
$$

\subsection{A Preliminary Study of Orbits}

a) Variation of ellipticity: It is clear from (36) that $\mathscr{H}>0$ implies that $E>0$. Stellar systems with positive total energy eventually disperse to infinity. Therefore $(x, y)$ should asymptotically increase without bound for $\mathscr{H}>0$. One such orbit is shown in Fig. 2 for $\mathscr{H}=0.1$. The orbit can be interpreted as a collapse from infinity followed by bounce and expansion back to infinity at a different ellipticity.

Under what circumstances will ellipticity be conserved? We should look for conditions under which $u$ is constant while $x$ and $y$ change with time; if $\dot{y} / \dot{x}=y / x$ at

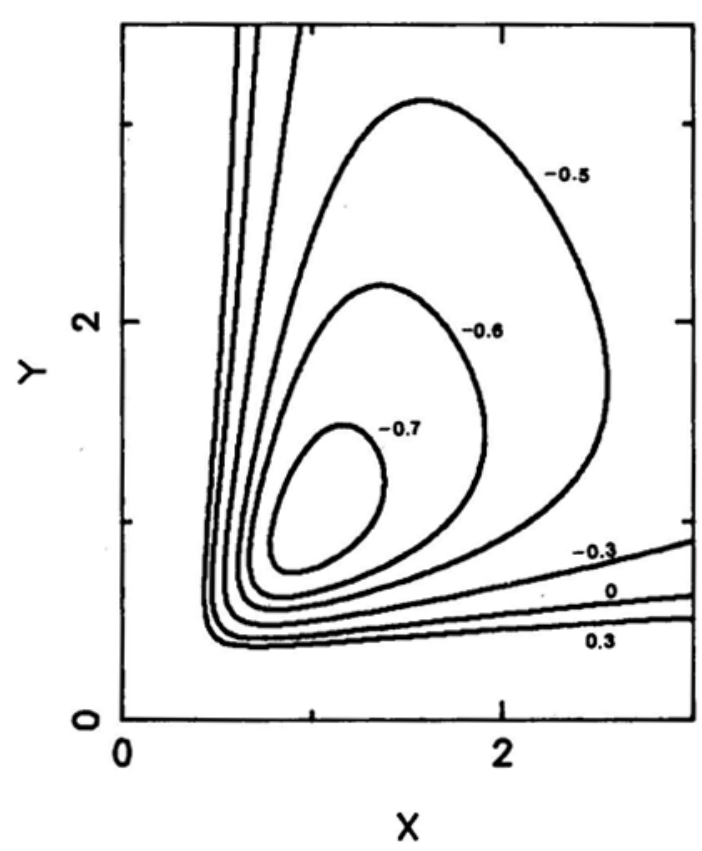

Figure 1. Level surfaces of $\mathscr{V}(\mathrm{x}, y)$ for $\alpha=1, \beta=3 / 2$. 


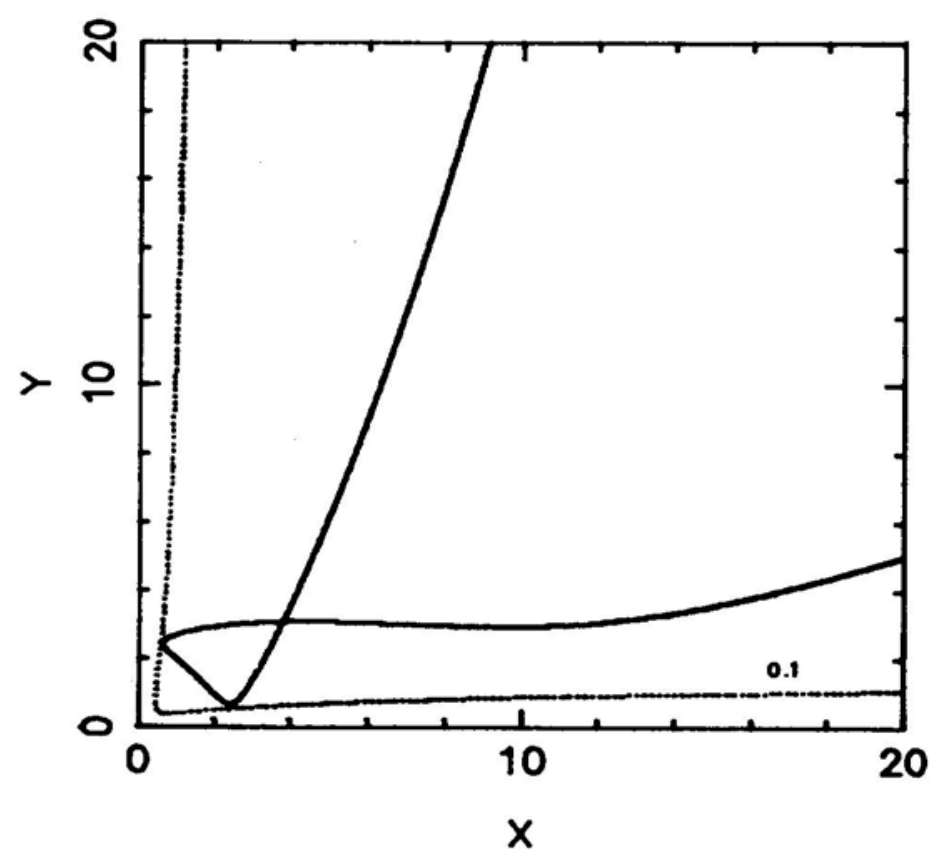

Figure 2. An unbounded orbit a $\mathscr{H}=0.1$ showing the changes in ellipticity.

Some time under what circumstances is $\ddot{y} / \ddot{x}=y / x$. Using (33) we require

$$
\frac{\alpha / y^{3}-2 \beta / x^{2}(1-u g)}{1 / x^{3}-\beta g / x^{2}}=u \text {. }
$$

Since $u$ is assumed to be constant, we can use (37) to set

$$
\alpha=2 u^{3}(1-u g) / g \text {. }
$$

Using the identity (34) for $g(u)$, we get the condition

$$
\left(1-u^{2}\right) \frac{\mathrm{d} g}{\mathrm{~d} u}=0 .
$$

$g$ is an increasing function of $u$. Therefore $u=1$; the only self-similar oscillations allowed are spherical oscillations. When $u=1, g=2 / 3$ and $\alpha=1$. Then (33) reduces to

$$
\ddot{x}+\frac{(2 \beta / 3)}{x^{2}}-\frac{1}{x^{3}}=0 .
$$

We determine $\beta$ from (33). Since $\alpha=1, \Omega_{\mathrm{o}}=\omega_{0}$ and $a_{1}=a_{3}$ from the very definitions of $\omega_{0}$ and $\Omega_{0}$ in terms of $a_{1}$ and $a_{3}$. Therefore $\beta=\sqrt{\omega_{0}} / g(1)=\frac{3}{2} \sqrt{\omega_{0}}$. Using this we get

$$
\ddot{x}+\frac{\sqrt{\omega_{0}}}{x^{2}}-\frac{1}{x^{3}}=0
$$

which is identical to Equation (1) as expected. 
b) General features: We have solved equations (33) numerically for $\alpha=1$ and $\beta=3 / 2$ using a simple first-order symplectic scheme (update momenta and then coordinates). The suitability of the scheme was tested on the Toda Hamiltonian (Lichtenberg \& Lieberman 1983) with satisfactory results-no spurious chaos induced by discretization or round-off was found. Fig. 3 shows a surface of section ( $\mathrm{p}_{y}$ versus $y$ at $x=1$, $p_{x}>0$ ) for $\mathscr{H}=-0.45$. The (unstable) fixed point on the upper left corner corresponds to oscillations of uniform spheres. For $\mathscr{H} \lesssim-0.45$, this fixed point is stable (although we do not show the section here). At $\mathscr{H} \simeq-0.45$, the oscillating sphere is unstable to spheroidal modes and bifurcates into a periodically oscillating spheroid. It should be noted that this is not necessarily a general feature of all uniform-density oscillating spheres. The class of uniform density spheroids is much more restricted than the class of uniform density spheres. So the present work allows us to note only that the instability occurs for the subset of spheres that are members of a sequence of uniform spheroids.

The stable fixed point on the right-hand side with its accompanying islands corresponds to oscillations that are roughly "orthogonal" to the oscillations of spheres (i.e. they represent oscillations of ellipticity). The chain of islands in between is due to orbits trapped near a 5:3 resonance.

Figure 4 is a section at $x=2.5, p_{x}>0$ for $\mathscr{H}=-0.3$. At this high value of the basic instability of the spheres has given birth to chaotic oscillations of spheroids. The oscillation "orthogonal" to spherical oscillations is still stable and large regions of phase space around this are filled with regular orbits.

\subsection{Discs and Needles}

We briefly discuss the extreme oblate/prolate limits of the spheroidal model. These correspond to discs and needles respectively.

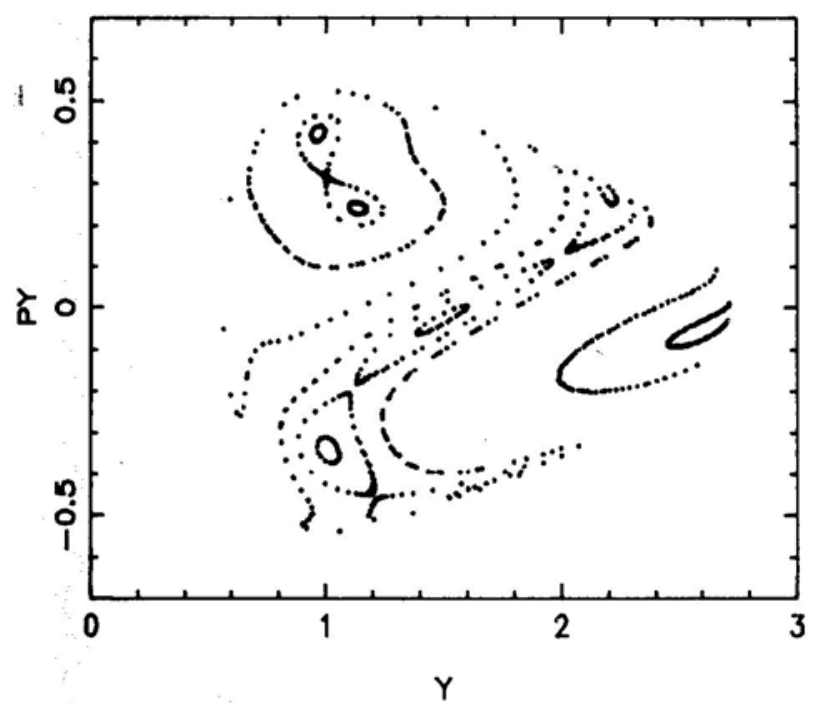

Figure 3. Surface of section $\left(x=1, \mathrm{p}_{x}>0\right)$ for $\mathscr{H}=-0.45$. 


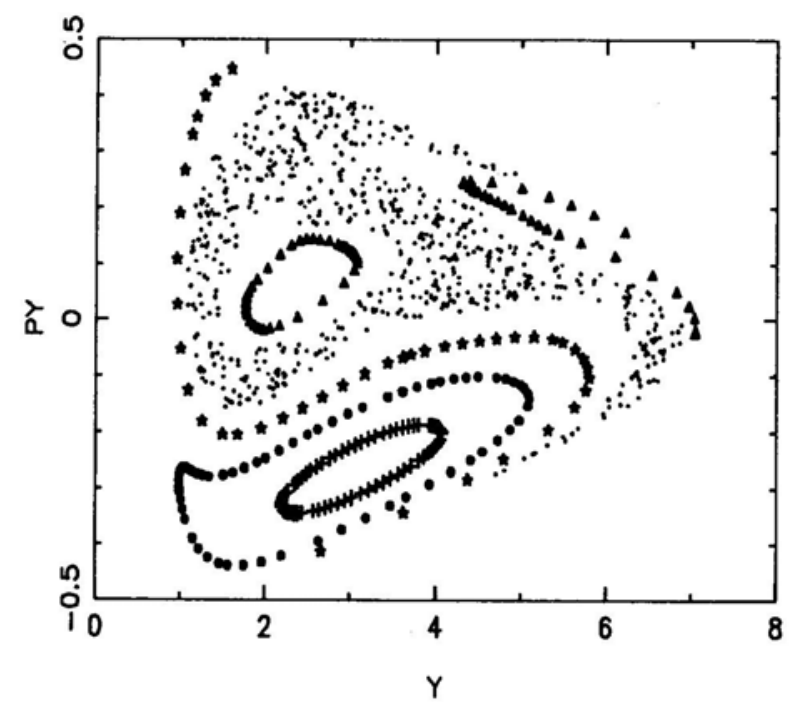

Figure 4. Surface of section $\left(\mathrm{x}=2.5, p_{x}>0\right)$ for $\mathscr{H}=-0.3$.

\section{a) Discs}

In the static model we let $a_{3} \rightarrow 0$, while keeping the mass $(M)$ fixed. The oscillations perpendicular to the disc are described by the time-dependent behaviour of $b_{3}$. Since we are looking for solutions that correspond to highly flattened configurations, we let $b_{3} \rightarrow 0$,

When

$$
a_{3} \rightarrow 0, \quad m=a_{3} / a_{1} \rightarrow 0 .
$$

Also $g(m)=\pi / 2$ and $\Omega_{0}^{2}=3 \pi G M / 4 \mathrm{a} 3 / 1$ in this limit. From Equation(32), $\beta$ $=\sqrt{\Omega_{0}} / g(m) \rightarrow 2 \Omega \Omega_{0} / \pi$. We recall that $b_{1}=\sqrt{\Omega_{0}} \alpha_{1} \chi$ and $b_{3}=\sqrt{\Omega_{0}} a_{1} y$. Since $b_{3} \rightarrow 0$, and both $\sqrt{\Omega_{0}}$ and $a_{1}$ are finite, $\mathrm{y} \rightarrow 0$. The oscillations in the plane of the disc are described by the first of the Equations (33):

$$
\ddot{x}+\frac{\beta}{x^{2}} g(y / x)-\frac{1}{x^{3}}=0 .
$$

Since $y \rightarrow 0$, we replace $g(y / x)$ by $g(0)=\pi / 2$ and write

$$
\ddot{x}+\frac{\sqrt{\Omega_{0}}}{x^{2}}-\frac{1}{x^{3}}=0
$$

which governs the oscillations of the radius $\left(b_{1}\right)$ of the disc with surface density $\propto$ $\left(1-\mathrm{r}^{2} / b^{2}\right)^{1 / 2}$ obtained by projecting a uniform density spheroid onto its plane of symmetry. 
b) Needles

This extremely prolate limit of spheroids corresponds to $\alpha_{1} \rightarrow 0$ in the static model, the mass of which is kept constant at $M$. When $a_{1} \rightarrow 0, m=a_{3} / a_{1} \rightarrow \infty$ and asymptotically

$$
b_{1}=\left(\frac{3 G M a_{3}}{2}\right)^{1 / 4} \frac{x}{\sqrt{m}}, \quad b_{3}=\left(\frac{3 G M a_{3}}{2}\right)^{1 / 4} \frac{y}{\sqrt{m}} .
$$

The equilibrium values of $x$ and $y$ are determined by setting $b_{1}=a_{1}$ and $b_{3}=a_{3}$ :

$$
x_{0}=\left(\frac{3 G M}{2 a_{3}^{3}}\right)^{-1 / 4} \frac{1}{\sqrt{m}}, \quad y_{0}=\left(\frac{3 G M}{2 a_{3}^{3}}\right)^{-1 / 4} \sqrt{m} \text {. }
$$

The oscillations of the length of the needle are described by the second of the Equations (33)

$$
\ddot{y}+\frac{2 \beta}{x^{2}}(1-u g)-\frac{\alpha}{y^{3}}=0 .
$$

Since the equilibrium value of $\mathrm{x} \rightarrow 0$ when $\mathrm{m}, \rightarrow \infty$ we set $u=y / x \simeq m$ in the above equation

$$
\ddot{y}+\frac{2 \beta}{y^{2}}\left[m^{2}(1-m g(m))\right]-\frac{\alpha}{y^{3}}=0 .
$$

As $m \rightarrow \infty$

$$
\begin{gathered}
\alpha=\frac{\omega_{0}^{2}}{\Omega_{0}^{2}}\left(\frac{a_{3}}{a_{1}}\right)^{4} \rightarrow 2 m^{2} \ln m \\
\beta=\sqrt{\Omega_{0}} / g(m) \rightarrow\left(\frac{3 G M}{2 a_{3}^{3}}\right)^{1 / 4} m^{3 / 2} \\
m^{2}(1-m g) \rightarrow \ln m, \\
\Omega_{0} \rightarrow\left(\frac{3 G M}{2 a_{3}^{3}}\right)^{1 / 2} m .
\end{gathered}
$$

Also, since $\mathrm{y} \rightarrow \infty$ we need to work with $b_{3}=\sqrt{\Omega_{0}} \alpha_{1} \mathrm{y}$. So, with $y=b_{3} / a_{1} \sqrt{\Omega_{0}}$, we have

$$
\ddot{b}+3 G M(\ln m)\left[\frac{1}{b_{3}^{2}}-\frac{a_{3}}{b_{3}^{3}}\right]=0 .
$$

This is the equation of motion for a particle in a potential well that is infinitely deep at $b_{3}=a_{3}$. Strictly speaking the original CBE is not applicable to 2 and lower dimensional systems (Rybicki 1972). The calculations on discs and needles given above continue to describe systems in which the thickness is finite but small compared to the other dimensions.

\section{Conclusions}

There has been earlier work on the oscillations of uniform spheres and spheroids by Chandrasekhar \& Elbert (1972, hereafter CE) and SomSunder \& Kochhar $(1985,1986$, 
hereafter SK I and SK II). The approach of CE was to apply the scalar form of the Virial theorem to a sphere. The moment of inertia term and the potential term could be expressed in terms of the instantaneous radius, $a$, and the kinetic energy followed from energy conservation. The resulting equation for the variation of $\alpha$ with time is identical to that derived in Paper I. Notice that application of the Virial theorem in this manner presupposes the existence of undamped oscillations, while the application of the Lewis invariant proves it. CE used the tensor Virial to study the oscillations of spheroidal systems. There are now two independent kinetic energy terms along the $a_{1}$ and $a_{3}$ axes, so energy conservation alone is insufficient. CE introduced an additional postulate setting these equal to each other at all times (an algebraic error was rectified by SK I). SK II criticized this assumption as unnatural and instead assumed that the mean Streaming velocity in the stellar system was a linear function of the coordinates. This hypothesis was a natural one to make sure that the uniform density and spheroidal shape are preserved as for fluid ellipsoids. This assumption enabled them to derive a pair of coupled equations for $a_{1}$ and $a_{3}$ which are identical to Equation (26) of this paper. This connection is to be expected since the Lewis invariant $I$ (Equation 4) depends on velocity in the combination $|\xi \mathbf{v}-\xi \mathbf{r}|^{2}$. When the distribution function depends on $v$ through it is clear that the mean value of $v$ is a linear function of $r$. In brief, the distribution functions presented in this paper provide underlying detailed dynamical models realizing the assumptions of CE for spheres and SK II for spheroids. We know of no way to provide a similar basis for general (e.g. Gaussian) density profiles studied in CE and SK II. We should also mention that the limiting case of a cold collapsing spheroid has been studied by Lin, Mestel \& Shu (1965).

The method presented in this paper can be used to construct a time-dependent sity $\sim\left(1-\frac{x^{2}}{a^{2}}-\frac{y^{2}}{b^{2}}\right)^{1 / 2}$ which gives rise to a potential that is quadratic and $x$ and $y$ The distribution function is a function of $E_{\mathrm{x}}$ and $E_{\mathrm{y}}$ So, replacing the energies by the Lewis invariants would give rise to a pair of coupled, second-order, autonomous, ordinary differential equations describing the oscillations of the axes. It is not clear whether similar generalization of Freeman's (1966b) homogeneous, triaxial ellipsoid is possible.

The stability of these oscillating solutions is an important question that remains unanswered. If a given oscillating solution is stable, it implies the existence of nearby solutions which do not have precisely uniform density, but share its nonrelaxing properties. We know that the stability of static models depends on the details of the distribution function - there is a trend for hotter models in general to be stable. When the parent static uniform sphere or spheroid is stable, one might expect models with sufficiently small oscillations to be stable as well. At present we only have numerical evidence (see Paper 1) for the stability of the oscillations of a system consisting of plane parallel sheets. The existence of nonrelaxing solutions would probably be missed by numerical codes which directly attack the CBE (e.g. White 1986) because of the unavoidable dissipation produced by finite grid size.

\section{Acknowledgement}

We would like to thank Dr R. K. Kochhar for pointing out the close relationship of this work to that in SK II. 


\section{References}

Binney, J., Tremaine, S. 1987, Galactic Dynamics, Princeton Univ. Press, New Jersey.

Chandrasekhar, S. 1969, Ellipsoidal Figures of Equilibrium, Yale Univ. Press, New Haven.

Chandrasekhar, S., Elbert, D.1972, Mon. Not .R. astr. Soc 155, 435.

Freeman, K. C. 1966b, Mon. Not. R. astr. Soc., 134, 15

Freeman, K. C. 1966a, Mon. Not. R. astr. Soc., 134, 1.

Fridman, A. M., Polyachenko, V. L. 1984, Physics of Gravitating Systems I, Springer-Verlag, New York.

Goldstein, H.1980, Classical Mechanics, 2nd Edn, Addison-Wesley, Singapore.

Lewis, H. R. 1968, J. math. Phys., 9, 1976

Lichtenberg, A. J., Lieberman, M. A. 1983, Regular and Stochastic Motion, Springer, New York.

Lin, C. C, Mestel, L, Shu, F. H. 1965, Astrophys. J., 142, 1431.

Rybicki, G. B. 1972, Gravitational N-Body Problem Ed. M. Lecar, D. Reidel, Dordrecht

SomSunder, G., Kochhar, R. K. 1985, Mon. Not. R. astr. Soc., 213, 381(SK I).

SomSunder, G., Kochhar, R. K. 1985, Mon. Not. R. astr. Soc., 221, 553(SK II).

Sridhar, S. 1989, Mon. Not. R. astr. Soc,. 238, 1159.

White, R. L. 1986, The Use of Supercomputers in Stellar Dynamics, Eds P. Hut \& S. McMillan, Springer-Verlag, New York. 